\title{
Over-the-counter suboptimal dispensing of antibiotics in Uganda
}

\author{
This article was published in the following Dove Press journal: \\ Journal of Multidisciplinary Healthcare \\ 19 August 2013 \\ Number of times this article has been viewed
}

\author{
Jackson K Mukonzo',2 \\ Proscovia M Namuwenge ${ }^{1}$ \\ Gildo Okure ${ }^{3}$ \\ Benjamin Mwesige \\ Olivia K Namusisi ${ }^{4}$ \\ David Mukanga ${ }^{4}$ \\ 'Center for Operational Research \\ Africa, Kampala, Uganda; ${ }^{2}$ Department \\ of Pharmacology and Therapeutics, \\ ${ }^{3}$ School of Public Health, Makerere \\ University, Kampala, Uganda; ${ }^{4}$ African \\ Field Epidemiologist Network, \\ Kampala, Uganda
}

Correspondence: Jackson K Mukonzo Department of Pharmacology and Therapeutics, College of Health Sciences, Makerere University, Kampala University Road, PO Box 7072, Kampala, Uganda Tel +256772593168

Email mukojack@yahoo.co.uk
Background: Overuse and misuse of antibiotics is a serious global problem. While resistance to older antibiotics is increasing, development of newer molecules has stalled. Resistance to the existing antibiotics that is largely driven by their high-volume use is a global public health problem. Uganda is one of the countries where prescription-only drugs, including antibiotics, can be obtained over the counter. We determined the rate of antibiotic dispensing and use in Uganda.

Methods: The study utilized a descriptive cross-sectional study design to determine the number of antibiotic "prescribed" daily doses per 1,000 clients. Data were collected from one health center II, eight general/district hospitals, one national referral hospital, and 62 registered community pharmacies. From each study site, data were collected for five consecutive days over the months of November 2011 to January 2012.

Results: The overall antibiotic issue rate was $43.2 \%$. Amoxicillin, metronidazole, ciprofloxacin, sulfamethoxazole-trimethoprim, cloxacillin, and ampicillin, belonging to the WHO anatomical therapeutic chemical classifications of penicillin with extended spectra, imidazole derivatives, fluoroquinolones, and sulfonamide-trimethoprim combinations, constituted $70 \%$ of the issued antibiotics. About $41 \%$ of antibiotics were issued over the counter. At community pharmacies, where $30 \%$ of antibiotic dispensing occurred, the number of prescribed daily doses/1,000 antibiotic clients was 4,169 compared to 6,220, 7,350 and 7,500 at general/district hospitals, the national referral hospital, and the health center, respectively.

Conclusion: In Uganda, at least four in every ten individuals that visit a health-care facility are treated with an antibiotic. Antibiotics are largely given as over-the-counter drugs at community pharmacies. The number of antibiotic prescribed daily doses/1,000 antibiotic clients does not significantly differ between categories of health-care facilities except at community pharmacies, where lower doses are dispensed compared to other health-care facilities.

Keywords: antibiotic, over-the-counter dispensing, suboptimal dosing, Uganda

\section{Introduction}

The inappropriate use of antibiotics for the treatment of patients with common infections is a worldwide problem, with implications for increasing treatment costs, adverse events, and selection of antibiotic-resistant germs. ${ }^{1}$ A significant amount of antibiotic misuse results from their indiscriminate accessibility. Prohibitive medical consultation costs and knowledge of the possibility to acquire antibiotics without prescription $^{2}$ facilitate their acquisition over the counter. ${ }^{1}$ Both inappropriate and suboptimal exposure to antibiotics constitutes their misuse and is in turn associated with bacterial resistance. ${ }^{3,4}$ The global antibiotic-resistance problem is increasing at varying rates 
in different regions. Countries with the most reserved antibiotic-prescribing patterns have relatively lower rates of antibiotic resistance. ${ }^{5}$

According to Avison, one of the ways to combat antibiotic resistance is simply to develop new drugs. ${ }^{6}$ Despite the rising need for newer antimicrobial drugs, there has been a several-decades-long gap in new-antibiotic development. ${ }^{7-9}$ This might be attributed to the likely rapid development of antibiotic resistance, sometimes resulting in loss of resources by pharmaceutical companies, the limited market for antibiotics since bacterial infections diseases are commonest among the poor, and the global restrictions on antibiotic use. Other ways to minimize the development of antibiotic resistance include limiting their use, discouraging their misuse, and reducing infectious disease burden through preventive practices. ${ }^{10}$ Despite the existence of policies regulating their use, antibiotics are readily available over the counter in many countries. ${ }^{11-15}$

In Uganda, a low-income country, public health is based upon a referral system, with village health teams as the lowest health-care structure, followed by health centers (II, III and IV), district hospitals, regional referral hospitals, and national referral hospitals. Each health center II, the lowest type of formal health-care facility, usually serves a few thousand people. It is normally led by an enrolled nurse, working with a midwife, two nursing assistants, and a health assistant, who are facilitated to offer antenatal care and treat common diseases like malaria and other common diseases on an outpatient basis. Patient care at health centers follows treatment protocols that highlight the disease symptoms, the recommended drugs, and their doses. District hospitals, on the other hand, are expected to offer comprehensive care, including a full range of surgical services. In the case of inability to handle a disease condition, district hospitals refer patients to regional referral hospitals, which may consequently refer to national referral hospitals.

The private health sector in Uganda constitutes hospitals whose operations are scaled at different levels up to that of regional referral hospital. It also constitutes community pharmacies that satisfy a criterion of the national licensing body. While health-service costs at public health-care facilities are paid by the government, the cost of service at private health-care facilities is largely paid out of pocket, without any subsidies from the government. Although health-care provision is guided by standard treatment guidelines, including dispensing antibiotics as "prescription-only-drugs" in Uganda, these are inadequately enforced. This together with several socioeconomic factors may facilitate indiscriminate access to antibiotics in the country. In this study, we established the rate of antibiotic dispensing and the prescribed daily doses for antibiotics at various health-care structure levels in Uganda.

The World Health Organization Collaborating Center in Oslo has developed the anatomical therapeutic chemical classification/defined daily dose (ATC/DDD) system to serve as a tool for drug-utilization research in order to improve the quality of drug use. The system has been used since the early 1970 s, and has been demonstrated to be suitable for national and international comparisons of drug utilization, evaluation of long-term trends in drug use, assessing the impact of certain events on drug use, and providing baseline data in investigations of drugs. ${ }^{16}$

\section{Materials and methods}

This is an explorative study that describes the practice of antibiotic dispensing in health-care facilities in Uganda. In the current study, the ATC/prescribed daily dose (PDD) per 1,000 recipients, a modification of the ATC/DDD, was used for presenting antibiotic-utilization statistics at various health-care facility categories in Uganda. The study utilized a descriptive cross-sectional design to determine the antibiotic PDD per 1,000 antibiotic recipients per day at individual study sites, as previously described. ${ }^{17,18}$ Quantitative methods of data collection were used. Structured data-collection forms were used to record information on antibiotics dispensed/ issued or prescribed to patients. The study was approved by the Uganda National Council for Science and Technology.

\section{Study sites and population}

The study was conducted in nine districts, including Kampala. The other eight districts - Kabarole, Busenyi, Masindi, Butaleja, Iganga, Kaabong, Arua, and Amolatar - were purposively selected to represent eight geographical regions of Uganda. Within each district, a hospital (private, nongovernmental organization, or government-owned) and a community pharmacy were selected as study sites (Table 1), except Kampala District, where 55 registered community pharmacies were randomly selected. Mulago National Referral Hospital was purposively selected to capture data from a referral-level health-care facility. Katooke Health Center II was randomly selected in order to capture data on antibiotic prescription/ dispensing at the lowest physical levels of Uganda's public health-care system. The study unit constituted an individual patient dispensing record. Noting that adult doses are usually prescribed/dispensed for 12-year-old or older patients, prescription or dispensing records were included if the patient was 12 or more years old. 
Table I The total number of study participants per health-care facility (HCF), facility category, and geographical location in Uganda

\begin{tabular}{|c|c|c|c|c|c|c|c|c|c|}
\hline \multirow[t]{2}{*}{ Region } & \multicolumn{2}{|c|}{ Community pharmacies } & \multicolumn{2}{|c|}{ National referral hospital } & \multicolumn{2}{|c|}{ District hospital } & \multicolumn{2}{|c|}{ Health center } & \multirow{2}{*}{$\begin{array}{l}\text { Total } \\
\text { patients }\end{array}$} \\
\hline & $\begin{array}{l}\text { Number } \\
\text { of HCFs }\end{array}$ & $\begin{array}{l}\text { Number of } \\
\text { patients }\end{array}$ & $\begin{array}{l}\text { Number } \\
\text { of HCFs }\end{array}$ & $\begin{array}{l}\text { Number } \\
\text { of patients }\end{array}$ & $\begin{array}{l}\text { Number } \\
\text { of HCFs }\end{array}$ & $\begin{array}{l}\text { Number } \\
\text { of patients }\end{array}$ & $\begin{array}{l}\text { Number } \\
\text { of HCFs }\end{array}$ & $\begin{array}{l}\text { Number } \\
\text { of patients }\end{array}$ & \\
\hline Kampala & 56 & 15,475 & I & $\mathrm{I}, 843$ & 0 & 0 & 0 & 0 & 17,318 \\
\hline Northern & 1 & 416 & 0 & 0 & 1 & 121 & 0 & 0 & 537 \\
\hline West Nile & 1 & 400 & 0 & 0 & I & 387 & 0 & 0 & 787 \\
\hline Northwest & I & 385 & 0 & 0 & I & 402 & 0 & 0 & 789 \\
\hline Western & I & 642 & 0 & 0 & 1 & 169 & 1 & 173 & 984 \\
\hline Southwest & I & 249 & 0 & 0 & 1 & 455 & 0 & 0 & 704 \\
\hline Eastern & 0 & 0 & 0 & 0 & I & 312 & 0 & 0 & 312 \\
\hline East central & 0 & 0 & 0 & 0 & I & 466 & 0 & 0 & 466 \\
\hline Central & I & 100 & 0 & 0 & I & 416 & 0 & 0 & 516 \\
\hline Total & 62 & 17,667 & I & $\mathrm{I}, 843$ & 8 & 2,728 & I & 173 & 22,411 \\
\hline
\end{tabular}

Note: Totals of both participants and health-care facilities are indicated.

\section{Sample size and sampling procedure}

The sample size for community pharmacies was based on the central systematic area sample (CSAS) principle, using a definite population assumption. Over all, about $650 \mathrm{com}$ munity pharmacies were, at the time of the study, registered to sell human drugs in Uganda. Assuming a coverage proportion of $50 \%$ and a precision of $\pm 10 \%, 62$ community pharmacies were required for the study. Considering that about $50 \%$ of all registered community pharmacies in Uganda are located in Kampala District, 55 of the 62 community pharmacies were selected from Kampala District on the basis of the CSAS principle. Seven other community pharmacies were randomly selected from districts other than Kampala that were included in the survey. Registered community pharmacies in Kampala District were stratified and randomized per division.

Following stratification of the country into eight geographical regions (northwestern, northern, west Nile, western, southwest, eastern, east central, and central) according to the national TB-HIV/AIDS service provider's map; general/ district hospitals in each region were listed in alphabetical order, and every second district hospital was systematically selected from each region. Mulago National Referral Hospital was purposively selected so as to determine antibiotic consumption at the highest level of the health-care system.

\section{Data collection, management, and analysis}

From each study site, data were collected for 5 consecutive days over the months of November 2011 to January 2012. Prescription/dispensing records of clients that visited the study sites during the study period were used to fill a preformulated and piloted data-collection form. Where verbal prescriptions had been given and dispensed, the research assistant used other forms of records maintained at the facility, such as receipt books, to fill in the data-collection form. Data was entered into Epi Info version 3.3.5 (CDC, Atlanta, GA, USA) and cleaned before analysis using Statistica version 7 (StatSoft, Tulsa, OK, USA) and SPSS version 12 (IBM, Armonk, NY, USA). The antibiotic PDDs per 1,000 antibiotic recipients were obtained by calculating the total amount in grams for each dose of antibiotic dispensed and dividing the total by the DDD as conversion factor, obtained from the ATC/DDD index 2011 (www.whocc.no/atcddd).

\section{Results}

A total of 51,501.7 PDDs were issued at the study sites over 5 days during the study. A total of 9,691 clients (43.2\%) of 22,411 who presented at the study sites over the same period received at least one antibiotic as part of their treatment (Table 1). Antibiotic-issue rates were 78.5\%, 68.2\%, 30\%, and $19.7 \%$ at the national referral hospital, general hospitals, community pharmacies, and health center, respectively. The health center recorded the highest number of antibiotic PDDs per 1,000 antibiotic clients $(7,500)$ followed by the national referral hospital $(7,350)$ and general hospitals $(6,320)$ (Table 2). Community pharmacies recorded the lowest number of antibiotic PDDs per 1,000 antibiotic clients $(4,169)$ (Figure 1); 50.2\% and 9.1\% were dispensed as outpatient and inpatient prescriptions, respectively, while $40.7 \%$ were dispensed over the counter.

Overall, 23 anatomical chemical classes of antibiotics were dispensed over the study period. The commonly dispensed categories of antibiotics (frequency $\geq 5 \%$ ) included penicillins with extended spectrum, $\beta$-lactamaseresistant penicillins, trimethoprim-and-sulfonamide combinations, macrolides, fluoroquinolones, and imidazole derivatives. Penicillins with extended spectra were dispensed at least once of every four occasions an antibiotic was dispensed, rendering them the most commonly dispensed 
Table 2 The total number of clients, number of clients that received an antibiotic, and the prescribed daily dose (PDD) per I,000 clients as well as per 1,000 antibiotic recipients by health-facility category

\begin{tabular}{lllll}
\hline Type of facility & $\begin{array}{l}\text { Total number } \\
\text { of clients }\end{array}$ & $\begin{array}{l}\text { PDD/I,000 } \\
\text { clients }\end{array}$ & $\begin{array}{l}\text { Number } \\
\text { of antibiotic clients }\end{array}$ & $\begin{array}{l}\text { PDD/I,000 } \\
\text { antibiotic clients }\end{array}$ \\
\hline All & 23,935 & $2,143.9$ & 9,69 I & 5,300 \\
National referral hospital & 1,843 & $5,746.8$ & $1,44 I$ & 7,350 \\
District/general hospital & 4,252 & $4,310.4$ & 2,900 & 6,320 \\
Health center & 173 & $1,474.0$ & 34 & 7,500 \\
Community pharmacy & 17,667 & $1,252.3$ & 5,307 & 4,169 \\
\hline
\end{tabular}

category of antibiotics, followed by trimethoprim-andsulfonamide combinations (16.8\%) and imidazole derivatives (15.7\%) (Table 3). At the individual drug level (level 3 of the ATC), amoxicillin, sulfamethoxazole-trimethoprim, metronidazole, ciprofloxacin, cloxacillin, and ampicillin were the most dispensed, at rates of $17.8 \%, 16.8 \%$, $15.5 \%, 8.7 \%, 7.5 \%$, and $6.8 \%$, respectively. Important to note is that although about 49 different antibiotics were dispensed during the study period, antibiotics other than the six most dispensed were each dispensed at a frequency less than $5 \%$.
The number of antibiotic PDDs per 1,000 antibiotic clients differed significantly between community pharmacies and the health center $(P<0.0001)$, district hospitals and national referral hospital $(P<0.0001)$, and the overall mean $(5,300, P=0.007)$. No differences were observed between district hospitals and the health center $(P=0.29)$ or the national referral hospital and the health center $(P=0.90)$. All facility-category PDD/1,000 antibiotic clients differed significantly from the overall mean PDD/1,000 antibiotic clients $(P<0.0001)$. Upon exclusion of community pharmacy data, the mean $\mathrm{PDD} / 1,000$

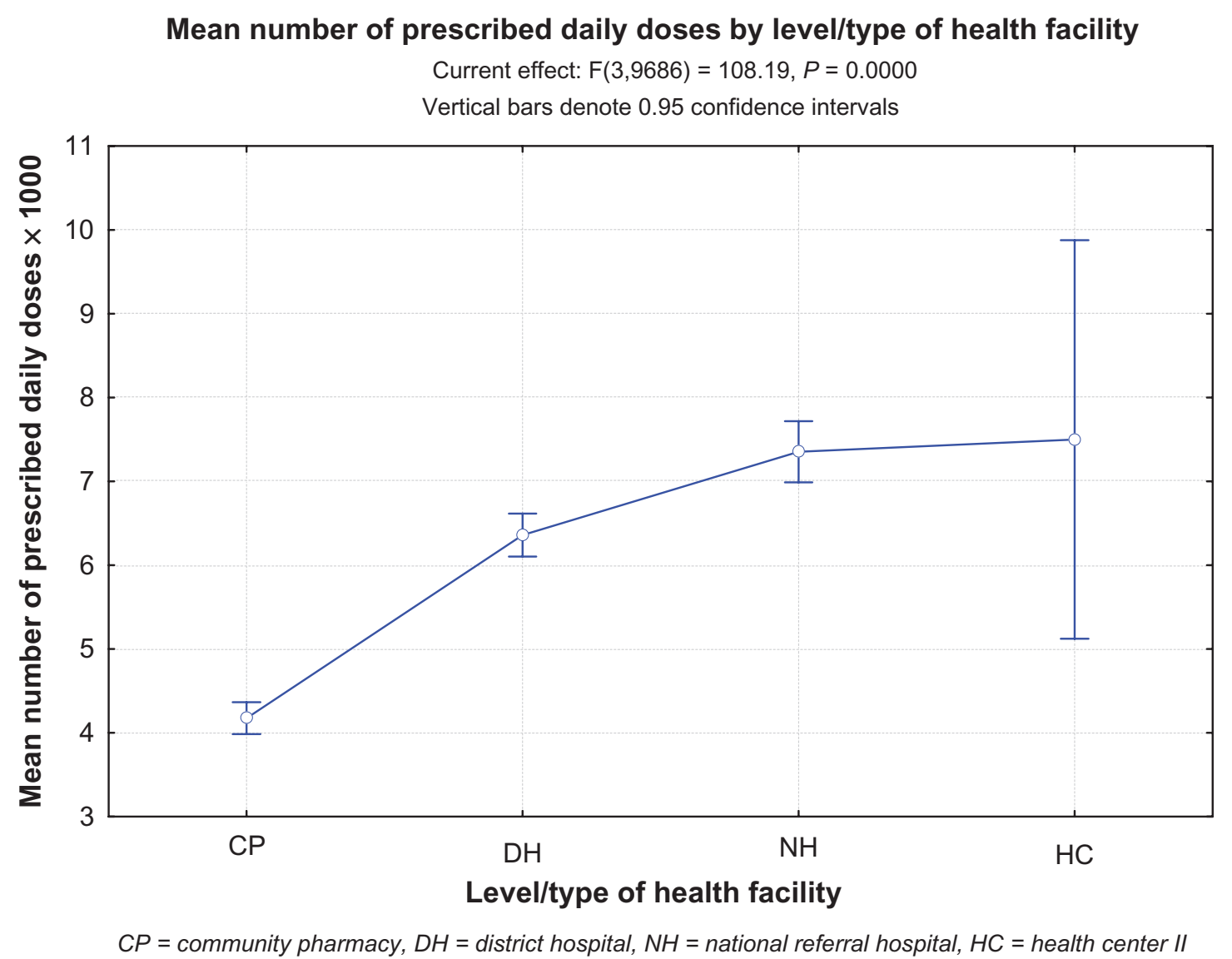

Figure I The mean number of prescribed daily doses per antibiotic recipient by health-care facility category or level. The plot compares number of prescribed daily doses/ I,000 between different health categories or levels in Uganda.

Abbreviations: $\mathrm{CP}$, community pharmacy; $\mathrm{DH}$, district hospital; $\mathrm{NH}$, national referral hospital; $\mathrm{HC}$, health center II. 
Table 3 Frequency of antibiotic dispensing by World Health Organization level II anatomical therapeutic chemical classification

\begin{tabular}{lll}
\hline Drug anatomical chemical class & Frequency & Percentage \\
\hline Penicillins with extended spectrum & 2,388 & 24.6 \\
Sulfonamides in combination with & 1,628 & 16.8 \\
trimethoprim & & \\
Imidazole derivatives & 1,518 & 15.7 \\
Fluoroquinolones & 985 & 10.2 \\
$\beta$-lactamase-resistant penicillins & 764 & 7.9 \\
Macrolides & 576 & 5.9 \\
Tetracyclines & 394 & 4.1 \\
Third-generation cephalosporins & 317 & 3.3 \\
Combinations of penicillins, including & 311 & 3.2 \\
$\beta$-lactamase inhibitors & & \\
$\beta$-lactamase-sensitive penicillins & 303 & 3.1 \\
First-generation cephalosporins & 140 & 1.4 \\
Amphenicols & 132 & 1.4 \\
Other aminoglycoside antibiotics & 121 & 1.2 \\
Nitrofuran derivatives & 36 & 0.4 \\
Second-generation cephalosporins & 23 & 0.2 \\
Other quinolones & 16 & 0.2 \\
Lincosamides & 9 & 0.1 \\
Drugs used to treat leprosy & 8 & 0.1 \\
Streptomycins & 7 & 0.1 \\
Others & 7 & 0.1 \\
\hline
\end{tabular}

antibiotic clients for the remaining facilities was 6,700 . This study revealed no significant differences in antibiotic number of PDDs per 1,000 antibiotic clients between privately owned, other than community pharmacies, and public health-care facilities (Figure 2).

\section{Discussion}

In this study, the rate of antibiotic use in Uganda was expressed in terms of both antibiotics issued per 100 drug recipients at dispensing windows and as the prescribed daily dose (PDD) per 1,000 antibiotic clients. $\mathrm{PDD} / 1,000$, which is based on the WHO's ATC/DDD system, ${ }^{16}$ is a relevant indicator to compare antibiotic use between different communities as well as health-care facilities. While DDD is the assumed average maintenance dose of a drug when used for its main indication in adults, PDD refers to total grams for each substance as given divided by grams, as defined by the WHO ATC/DDD definition. ${ }^{17}$ The calculated $\mathrm{PDD} / 1,000$ antibiotic recipients was, in this study, used as an indicator for the size of antibiotic dose issued at individual health-care facility categories, while the proportion of antibiotic issues indicated the rate of antibiotic use.

\section{Mean number of prescribed daily doses by type health facility ownership \\ Current effect: $F(2,9687)=138.57, P=0.0000$ \\ Vertical bars denote 0.95 confidence intervals}

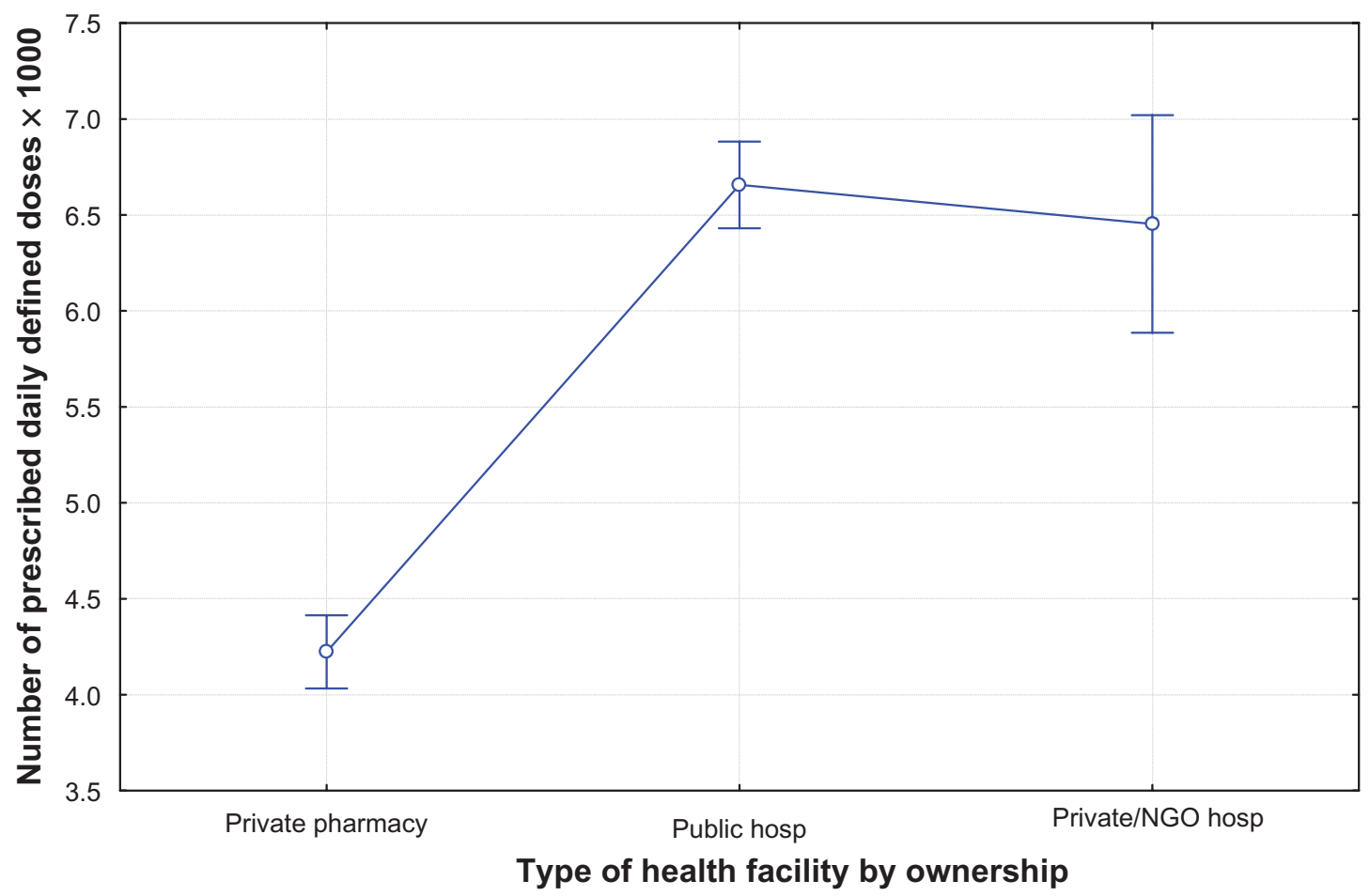

Figure 2 Number of prescribed daily doses/I,000 antibiotic recipients by health-facility ownership status. The graph demonstrates a significant difference between the community pharmacies and the rest of the health-care facilities, but no difference by ownership between public and privately owned health-care facilities. Abbreviation: NGO, nongovernmental organization. 
Inclusion of a wide breadth of health facilities in Uganda's health-care structure, ranging from the health center to the national referral hospital, as well as their regional distribution within the country, enables generalizability of the findings of this study.

Overall, the study reports a high antibiotic-issue rate of $43.2 \%$. In agreement with previous findings by a study conducted in Nigeria, $40.7 \%$ of all issues were over the counter at community pharmacies, ${ }^{19}$ which are also characterized by low PDD/1,000 antibiotic clients. Uganda's existing druguse regulatory policies prohibit over-the-counter dispensing of antibiotics. This together with the fact that community pharmacies mainly provide ambulatory health care might explain their lower antibiotic-issue rate. ${ }^{20}$ The generally reported high antibiotic-issue rate within the country might be attributed to (1) the predominance of empiric therapy that is largely due to inadequate laboratory facilities, the high frequencies of comorbidities, insufficient knowledge on the part of prescribers, and the high prescriber-patient ratios, and (2) endemicity of infectious disease. Infectious diseases, particularly those of bacterial cause, constitute Uganda's greatest burden of disease. This has been heightened by HIV/AIDS. As a result, antibacterial agents such as sulfamethoxazole-trimethoprim (cotrimoxazole) are often issued. Cotrimoxazole, which according to this study is issued once in every six occasions of antibiotic dispensing, is a constituent of the basic-care package for HIV patients, principally as a prophylactic agent against Pneumocystis carinii infections, which is a probable explanation for its high issue rate in Uganda.

Although determination of the relationship between over-the-counter antibiotic dispensing and underdosing was outside the scope of this study, the concurrently reported high rates of over-the-counter antibiotic dispensing and low doses, as well as corresponding variability in both measures between health-care facility categories, may reflect an association between antibiotic over-the-counter dispensing and underdosing, as reported previously. ${ }^{19}$ Over-the-counter antibiotic dispensing might be attributed to nonsuitability as well as enforcement gaps in drug-regulatory policies. Both over-the-counter sale of antibiotics ${ }^{21}$ and knowledge of the possibility to acquire antibiotics ${ }^{2}$ without a prescription are among the known determinants for self-medication with antibiotics in low-income countries, thus complicating the problem even further in such settings.

Comparable to what has been reported by a study conducted in similar settings, 49 drugs constituted the spectra of antibiotics dispensed during the study period..$^{22}$ The most dispensed antibiotics (constituting $70 \%$ of the most dispensed antibiotics) were amoxicillin, metronidazole, ciprofloxacin, sulfamethoxazole-trimethoprim, cloxacillin, and ampicillin. It was noted that at the time of the study, Mulago Hospital, Uganda's biggest national referral hospital, was equipped to perform sensitivity tests for only two (cotrimoxazole and ampicillin) of the six most dispensed drugs reported by this study. Other possible sensitivity tests at Mulago Hospital included rifampicin, amoxicillin-clavulanic acid, cefuroxime, flucloxacillin, tetracycline, vancomycin, penicillin $\mathrm{G}$, ceftriaxone, clindamycin, and nalidixic acid. Although the study did not establish antibiotic-sensitivity patterns, indiscriminate use of the drugs may lead to both wastage and treatment failures.

Suboptimal dosing is a major form of antibiotic misuse and has been associated with drug resistance. The significantly low antibiotic PDD/1,000 at community pharmacies compared to other health-care facilities might imply that patients who receive antibiotics from community pharmacies are more likely to receive suboptimal doses. Conversely, a possible effect of the small number of clients on the PDD/1,000 reported at the health center II may not be excluded. However, we do note that the highest PDD/1,000 registered at the health center is unlikely to have otherwise fallen out of range of the PDDs/1,000 registered at the national referral and district/general hospitals. Sublethal antibiotic exposures do not only expand the repertoire of genes that undergo selective pressures for the specific antibiotic causing the stress response $\mathrm{e}^{23}$ but have also been demonstrated to increase the horizontal transmission of mobile genetic elements among bacteria. ${ }^{24,25}$ In some organisms, low levels of bactericidal antibiotics have been shown to induce competence for transformation, leading to increased transfer and distribution of antibiotic-resistance genes. This together with the high antibiotic over-the-counter dispensing at community pharmacies raises a lot of concern, although it is worth noting that community pharmacies offer patients the flexibility of procuring drugs in small quantities until dosages are completed. The practice is, however, challenged by the fact that community pharmacies do not have the means or the capacity to track patient treatments. Whether or not community pharmacy staff comprehend the implications of inadequate antibiotic doses was beyond the scope of this study. However, we plausibly hypothesize that lower-antibiotic-dose dispensing at community pharmacies is market- rather than knowledge-driven, since all licensed pharmacies employ at least one qualified pharmacist who is charged with supervision of all drug-dispensing activities. 
Notably, other than at community pharmacies, the study reported no significant variation of $\mathrm{PDD} / 1,000$ at different health-care facilities from the mean value (Figure 2). This indicates that antibiotic use at health centers and hospitals may be predicted using data from any health-care facility category within the health-care structure in Uganda.

Notwithstanding the limitations of this study, which include low participation of some health-care facility categories, eg, health centers and national referral hospitals, lack of information on reasons for which care was sought, lack of antibiotic sensitivity data and dispenser and prescriber characteristics, the results of this study highlight the possible need for policy reforms on antibiotic dispensing, use, and monitoring in Uganda.

\section{Conclusion and recommendations}

In Uganda, at least four in every ten individuals who visit a health-care facility are treated with an antibiotic. Antibiotics are largely given as over-the-counter drugs at community pharmacies. The antibiotic PDD/1,000 antibiotic clients do not significantly differ between categories of health-care facilities except at community pharmacies, where lower doses are dispensed compared to other health-care facilities. While there is need to assess the reasons for which antibiotics are prescribed/issued at health-care facilities in Uganda, the results from this study indicate the need to address the problem of over-the-counter antibiotic dispensing at community pharmacies. Additionally, a comprehensive national strategy to contain the likely antimicrobial resistance in Uganda may be necessary.

\section{Disclosure}

The authors report no conflicts of interest in this work.

\section{References}

1. Cars O, Högberg LD, Murray M, et al. Meeting the challenge of antibiotic resistance. BMJ. 2008;337:a1438.

2. Grigoryan L, Burgerhof JG, Degener JE, et al. Determinants of selfmedication with antibiotics in Europe: the impact of beliefs, country wealth and the healthcare system. JAntimicrob Chemother. 2008;61(5): $1172-1179$.

3. Hawkey PM. The origins and molecular basis of antibiotic resistance. BMJ. 1998;317(7159):657-660.

4. Wise R, Hart T, Cars O, et al. Antimicrobial resistance. Is a major threat to public health. BMJ. 1998;317(7159):609-610.

5. Goossens H, Ferech M, Vander Stichele R, Elseviers M. Outpatient antibiotic use in Europe and association with resistance: a cross-national database study. Lancet. 2005;365(9459):579-587.

6. Avison MB. New approaches to combating antimicrobial drug resistance. Genome Biol. 2005;6(13):243.
7. Spellberg B, Talbot GH, Brass EP, Bradley JS, Boucher HW, Gilbert DN Position paper: recommended design features of future clinical trials of antibacterial agents for community-acquired pneumonia. Clin Infect Dis. 2008;47 Suppl 3:S249-S265.

8. Boucher HW, Talbot GH, Bradley JS, et al. Bad bugs, no drugs: no ESKAPE! An update from the Infectious Diseases Society of America. Clin Infect Dis. 2009;48(1):1-12.

9. Pechere JC, Hughes D, Kardas P, Cornaglia G. Non-compliance with antibiotic therapy for acute community infections: a global survey. Int J Antimicrob Agents. 2007;29(3):245-253.

10. Weinstein RA. Controlling antimicrobial resistance in hospitals: infection control and use of antibiotics. Emerg Infect Dis. 2001;7(2):188-192.

11. Shankar PR, Partha P, Shenoy N. Self-medication and non-doctor prescription practices in Pokhara valley, Western Nepal: a questionnairebased study. BMC Fam Pract. 2002;3:17.

12. Bin Abdulhak AA, Altannir MA, Almansor MA, et al. Non prescribed sale of antibiotics in Riyadh, Saudi Arabia: a cross sectional study. BMC Public Health. 2011;11:538.

13. Al-Faham Z, Habboub G, Takriti F. The sale of antibiotics without prescription in pharmacies in Damascus, Syria. J Infect Dev Ctries. 2011;5(5):396-399.

14. Plachouras D, Kavatha D, Antoniadou A, et al. Dispensing of antibiotics without prescription in Greece, 2008: another link in the antibiotic resistance chain. Euro Surveill. 2010;15(7):19488.

15. Grigoryan L, Monnet DL, Haaijer-Ruskamp FM, Bonten MJ, Lundborg S, Verheij TJ. Self-medication with antibiotics in Europe: a case for action. Curr Drug Saf. 2010;5(4):329-332.

16. Monnet DL, Mölstad S, Cars O. Defined daily doses of antimicrobials reflect antimicrobial prescriptions in ambulatory care. J Antimicrob Chemother. 2004;53(6):1109-1111.

17. de With K, Bestehorn H, Steib-Bauert M, Kern WV. Comparison of defined versus recommended versus prescribed daily doses for measuring hospital antibiotic consumption. Infection. 2009;37(4):349-352.

18. Hutchinson JM, Patrick DM, Marra F, et al. Measurement of antibiotic consumption: a practical guide to the use of the Anatomical Therapeutic Chemical classification and Defined Daily Dose system methodology in Canada. Can J Infect Dis. 2004;15(1):29-35.

19. Esimone CO, Nworu CS, Udeogaranya OP. Utilization of antimicrobial agents with and without prescription by out-patients in selected pharmacies in South-eastern Nigeria. Pharm World Sci. 2007;29(6): 655-660.

20. Konde-Lule J, Gitta S, Okuonzi S, Matsiko CW. Access to health care in rural Uganda. Presented at: iHEA 2007 6th World Congress: Explorations in Health Economics; July 8-11, 2007; Copenhagen, Denmark.

21. Lansang MA, Lucas-Aquino R, Tupasi TE, et al. Purchase of antibiotics without prescription in Manila, the Philippines. Inappropriate choices and doses. J Clin Epidemiol. 1990;43(1):61-67.

22. Palcevski G, Ahel V, Vlahovic-Palcevski V, Ratchina S, RosovicBazijanac V, Averchenkova L. Antibiotic use profile at paediatric clinics in two transitional countries. Pharmacoepidemiol Drug Saf. 2004;13(3):181-185.

23. Kohanski MA, DePristo MA, Collins JJ. Sublethal antibiotic treatment leads to multidrug resistance via radical-induced mutagenesis. Mol Cell. 2010;37(3):311-320.

24. Maiques E, Ubeda C, Campoy S, et al. Beta-lactam antibiotics induce the SOS response and horizontal transfer of virulence factors in Staphylococcus aureus. J Bacteriol. 2006;188(7):2726-2729.

25. Ubeda C, Maiques E, Knecht E, Lasa I, Novick RP, Penades JR. Antibiotic-induced SOS response promotes horizontal dissemination of pathogenicity island-encoded virulence factors in staphylococci. Mol Microbiol. 2005;56(3):836-844. 


\section{Publish your work in this journal}

The Journal of Multidisciplinary Healthcare is an international, peerreviewed open-access journal that aims to represent and publish research in healthcare areas delivered by practitioners of different disciplines. This includes studies and reviews conducted by multidisciplinary teams as well as research which evaluates the results or conduct of such teams or

healthcare processes in general. The journal covers a wide range of areas and welcomes submission from practitioners at all levels, from all over the world. The manuscript management system is completely online and includes a very quick and fair peer-review system. Visit http://www.dovepress.com/testimonials.php to read real quotes from published authors.

Submit your manuscript here: http://www.dovepress.com/journal-of-multidisciplinary-healthcare-journal 